\title{
Les Gens du voyage en France : représentations collectives et contrôle d'une minorité nationale
}

The Gypsy community in France: collective representations and control of a national minority

\section{Céline Bergeon}

\section{OpenEdition}

Journals

\section{Édition électronique}

URL : http://journals.openedition.org/belgeo/14864

DOI : 10.4000/belgeo.14864

ISSN : 2294-9135

Éditeur :

National Committee of Geography of Belgium, Société Royale Belge de Géographie

Référence électronique

Céline Bergeon, «Les Gens du voyage en France : représentations collectives et contrôle d'une minorité nationale », Belgeo [En ligne], 4 | 2014, mis en ligne le 20 décembre 2014, consulté le 19 avril 2019. URL : http://journals.openedition.org/belgeo/14864 ; DOI : 10.4000/belgeo.14864

Ce document a été généré automatiquement le 19 avril 2019

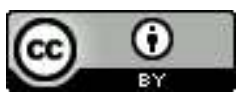

Belgeo est mis à disposition selon les termes de la licence Creative Commons Attribution 4.0 International. 


\title{
Les Gens du voyage en France: représentations collectives et contrôle d'une minorité nationale
}

\author{
The Gypsy community in France: collective representations and control of a \\ national minority
}

Céline Bergeon

1 Depuis les événements survenus pendant l'été 2009 en France suite au conflit opposant les forces de l'ordre à un groupe de Gens du voyage, le gouvernement français n'a cessé de renforcer ses contrôles et ses activités de surveillance. Ce climat répressif, dénoncé par les associations françaises, fait aussi l'objet de nombreux avertissements émis par le Conseil de l'Europe qui invite le gouvernement français à mieux vivre avec sa communauté des Gens du voyage et à stopper toute pratique discriminatoire concernant leur accès au droit commun. La situation française - comme beaucoup d'autres en Europe de l'Ouest - est complexifiée par un amalgame fort entre ceux que l'administration désigne comme Gens du voyage et la présence de Roms migrants originaires des pays de l'Europe de l'Est. Si les premiers, arrivés en France depuis le $14^{\text {ème }}$ siècle, sont des citoyens français et soumis à la législation nationale, les Roms migrants sont quant à eux dépendants des politiques migratoires mises en place au niveau européen, déclinées et adaptées au droit interne. Si une origine indienne commune - très souvent méconnue par ces deux groupes - serait leur point commun, ils demeurent néanmoins dans des situations très différentes concernant leur statut en France.

2 Cet article concerne exclusivement les Gens du voyage français. Il ne traite donc pas de la situation des Roms migrants en France, qui sont pour la plupart des citoyens roumains et bulgares. Ces familles migrantes sont touchées par une précarité résidentielle importante : elles vivent, pour beaucoup d'entre elles, dans des squats et des campements illégaux localisés dans les marges urbaines. Ces lieux de vie, très souvent insalubres, sont régulièrement démantelés par les autorités locales qui, dans le même temps, engagent 
des procédures d'expulsion du territoire français. Ainsi, sur le premier semestre 2013 en France, environ 10174 Roms migrants ont été expulsés de leur lieu de vie selon Amnesty International. Ces expulsions sont couramment relayées par la presse et font l'objet de débats animés au sein de la classe politique française comme dans les discussions citoyennes.

Les Gens du voyage sont donc des citoyens français qui ont un mode de vie circulant. Pour beaucoup d'entre eux, ils habitent en caravane et possèdent une organisation familiale très resserrée. En effet, la mobilité se vit en famille et dépend de différents critères (économiques, sociaux et culturels). Chaque membre de la famille contribue à l'équilibre familial par ses activités économiques et par les réseaux qu'il possède. À l'opposé, la circulation comme pratique sociale issue de plusieurs générations est considérée comme un moteur pour l'unité familiale. Les pratiques de mobilité des familles de Gens du voyage en France ont toujours été considérées comme problématiques, notamment par les acteurs politiques et les autorités policières. Différentes législations ont vu le jour depuis le début du $20^{\text {ème }}$ siècle dans l'objectif de mieux contrôler leur circulation. Cependant, ces procédures de surveillance ne sont pas spécifiques au cas français, le contrôle social opéré envers ces familles circulantes est un dénominateur commun à de nombreux pays européens comme l'Angleterre par exemple (Richardson, 2006).

Dans un premier temps, cet article se propose d'examiner les catégories existantes en France pour dénommer, identifier les Gens du voyage et pour encadrer leurs pratiques de mobilité. Ces catégories administratives sont mises en comparaison avec les désignations endogènes (Liégeois, 2009; Okely, 1983). Dans un deuxième temps, si les politiques publiques françaises visent le droit commun, des lois spécifiques sont pourtant mises en place pour les Gens du voyage posant ainsi la question délicate des limites entre droit commun et droit spécifique mais également de la reconnaissance des minorités nationales, normalement absente du droit français (Boev, 2011, p. 89). Cependant, les dispositifs législatifs particuliers qui visent notamment la circulation des Gens du voyage montrent clairement que les caractéristiques culturelles sont un critère de choix dans l'élaboration de différents textes, et ce depuis de nombreuses années. Ces différentes lois ont d'importantes conséquences sur les pratiques de mobilité des Gens du voyage : dans une dernière partie nous analyserons l'impact de la contrainte législative sur le quotidien des familles et nous mettrons en évidence les adaptations développées par les familles pour perpétuer leur mode de vie. Si les discours politiques soulignent une tendance à la sédentarisation des Gens du voyage, la recherche académique quant à elle pointe, au contraire, de nouveaux comportements circulatoires en réponse à la contrainte législative (Bergeon, 2011 ; Bordigoni, 2007).

5 Les résultats présentés dans ce papier proviennent d'un travail doctoral réalisé entre 2006 et 2011. L'objectif principal de cette recherche était d'étudier les impacts de dispositifs législatifs particuliers sur la circulation des familles de Gens du voyage en France. Ce travail a combiné plusieurs méthodologies de terrain dont des entretiens qualitatifs et une collecte de données concernant les parcours de familles, qui ont été par la suite cartographiés. Au total, ce sont 27 entretiens qui ont été passés auprès de différents profils de Gens voyage en termes de pratiques de mobilité et de logement. Les entretiens ont été conduits dans la région Poitou-Charentes en France $(7$ dans le département de la Vienne, 7 dans les Deux-Sèvres, 5 en Charente, et 8 en CharenteMaritime). Ce choix géographique - la région Poitou-Charentes - se justifie par une forte attractivité de ce territoire pour les familles de Gens du voyage grâce à sa diversité 
d'activités professionnelles saisonnières (tourisme, agriculture, etc.). La plupart des entretiens ont été possibles avec l'aide des associations locales qui travaillent auprès des Gens du voyage ; elles ont été d'une ressource formidable pour construire une relation de qualité et de confiance avec les Gens du voyage.

\section{Les catégorisations administratives au regard des identifications quotidiennes}

\section{Des catégorisations ethniques pour un pays qui refuse les connotations ethniques}

Depuis le début du $20^{\text {ème }}$ siècle, différentes dénominations ont été utilisées pour identifier la population des Gens du voyage en France. Cette évolution dans l'usage des termes est particulièrement visible à travers les textes législatifs, mettant ainsi en évidence la place singulière de cette population (Simhandl, 2005) dans la société française. Même si les connotations ethniques sont interdites par la Constitution française, l'ensemble de ces dénominations administratives se réfère implicitement au mode de vie circulant des Gens du voyage.

$7 \mathrm{Au}$ cours du $20^{\text {ème }}$ siècle, le premier texte législatif qui identifie spécifiquement la population des Gens du voyage date du 16 juillet 1912, et réglemente l'exercice des professions ambulantes et la réglementation de la circulation des nomades. Un paragraphe entier s'adresse aux populations qui « circulent sur le territoire français sans domicile ni résidence, n'exerçant pas de profession ou de commerce ambulant, ni la profession de commerçant ou d'industriel forain ». Cette loi instaurait, pour chaque "nomade », l'obligation de posséder un carnet anthropométrique sur lequel figuraient données biographiques, empreintes ou encore mensurations. Réel «passeport de l'intérieur " (Robert, 2007), le carnet anthropométrique devient un moyen de contrôle efficace pour les autorités françaises qui considéraient les « nomades » comme un danger du fait de leur mobilité spatiale parfois très soutenue. Ceux-ci étaient alors contraints de présenter leur carnet à chaque arrivée et à chaque départ d'une commune. Un visa était apposé par la Préfecture ou la Police, les autorisant ainsi à s'installer dans la municipalité. Au-delà de contrôles récurrents et gênants au quotidien, un des effets de cette disposition concerne directement la pérennité de la mobilité devenue très compliquée du fait de cet outil de contrôle. Leur installation dépendait uniquement de l'obtention du visa. La mobilité spatiale des familles de Gens du voyage étant intimement liée à l'exercice des activités économiques, un refus de visa à l'installation avait des conséquences importantes sur les ressources de la famille et donc sur leur capacité financière à continuer le voyage.

Cette loi a considérablement influencé l'opinion publique au début du $20^{\text {ème }}$ siècle. Les dispositions spécifiques prises envers les «nomades» ont érigé des pratiques quotidiennes en spécificités culturelles (Powell, 2008) et ont largement contribué au maintien voire à la généralisation de représentations négatives contribuant ainsi au renforcement des préjugés et autres idées reçues: «Roma and Gipsies-Travellers are recurrent objects of institutional regulation » (Brancroft, 2005, p. 33). Le texte de 1912 laisse place en 1969 à la loi n ${ }^{\circ}$ 69-3 du 3 janvier relative à l'exercice des activités ambulantes et au régime applicable aux personnes circulant en France sans domicile ni 
résidence fixe. Les nomades y sont, à nouveau, visés. Si certains considèrent cette loi comme un pas vers la " compréhension » (le carnet anthropométrique est supprimé), elle exige néanmoins que les «nomades" présentent de nouveaux documents administratifs. La loi du 3 janvier 1969 établit un parallèle manifeste entre les «nomades» et les catégories professionnelles ambulantes, puisqu'elle concerne :

- les commerçants ambulants, dont les activités doivent régulièrement être déclarées à l'administration. Cette catégorie de personne détient un « livret spécial de circulation ».

- les caravaniers, catégorie qui concerne les personnes logeant dans des résidences mobiles mais ayant des revenus réguliers. Il leur est délivré « un livret de circulation ».

- les nomades, dont l'habitat est mobile et les revenus irréguliers, et qui doivent faire viser leur «carnet de circulation" tous les 3 mois. Ce dernier remplace le carnet anthropométrique et permet à cette catégorie de jouir de droits sociaux. À la demande de ce titre de circulation, ils sont directement enregistrés dans un fichier central. Il leur est également demandé de choisir une commune de rattachement, afin de "profiter des avantages liés au domicile " tels que le mariage, la sécurité sociale ou encore le droit de vote. Ce rattachement prend acte pour deux ans.

9 Les associations françaises ont toujours considéré ces documents (carnet anthropométrique puis carnet de circulation) comme un acte discriminant envers une partie de la population française. Grâce à ces différentes mesures, la mobilité spatiale des Gens du voyage fait l'objet d'un contrôle continu alors que la liberté de circulation est écrite dans la Constitution française et constitue un des droits fondamentaux de chaque citoyen. L'ensemble de ces dispositifs n'a fait que contribuer à l'appauvrissement de pratiques de mobilité qui fédèrent pourtant un mode de vie. Ce n'est qu'en octobre 2012 que le Conseil Constitutionnel français a déclaré invalide deux des principales dispositions liées au carnet de circulation. Ainsi, l'obligation du visa est abrogée car considérée comme contraire à la liberté de circulation. De plus, les jeunes issus de la communauté des Gens du voyage peuvent désormais voter dès 18 ans alors qu'auparavant ils ne pouvaient le faire qu'à partir de 19 ans (le carnet est obligatoire à partir de 16 ans et le droit de vote était octroyé à partir de deux ans de possession de carnet).

10 La catégorie administrative «Gens du voyage » devient la désignation légale à partir des années 1990 en France. Un paragraphe concerne spécifiquement leur accueil à l'échelle locale dans un texte traitant de l'accès au logement des citoyens français. Les autorités compétentes suggèrent aux communes de s'équiper pour le stationnement des Gens du voyage. Simples recommandations, peu de municipalités - hormis quelques-unes notamment dans l'ouest de la France - ont réalisé des équipements en ce sens. Comme le terme de "nomade», la désignation «Gens du voyage» se réfère implicitement notamment avec le terme de "voyage » - à leur mode de vie et à leur mobilité spatiale. Ainsi, cette catégorie est construite " pour occulter officiellement l'ethnicité des groupes qu'elle désigne, pour lui substituer le préjugé ancestral de groupe marginal, délinquant, criminel » (Blum le Coat, Catarino, Quiminal, 2004, p. 163). L'ensemble de ces catégories contribue à créer une assignation identitaire comme si les pratiques quotidiennes et les modes de vie étaient figés.

11 Les Gens du voyage ont toujours été associés - par le biais d'un paragraphe - à des textes législatifs de plus grande ampleur comme ce fut le cas en 1912 et 1969 (associés aux activités ambulantes marchandes) et en 1990, visant la mise en œuvre du droit au logement. Une importante évolution marque le début des années 2000 avec la loi relative à l'accueil et à l'habitat des Gens du voyage, texte qui leur est exclusivement consacré. Il 
concerne en grande partie les dispositions relatives à l'accueil et au stationnement des familles à l'échelle locale qui deviennent, cette fois-ci, obligatoires. En effet, les communes de plus de 5000 habitants sont obligées d'encadrer le stationnement et d'accueillir les familles qui souhaitent faire une halte (plus ou moins prolongée) sur leur territoire. Ces équipements, appelés aire d'accueil, sont mentionnés dans un schéma départemental d'accueil des Gens du voyage. Celui-ci précise la localisation, les capacités, les équipements des aires d'accueil, lieux où les Gens du voyage doivent, en contrepartie, stationner. Le stationnement en dehors de ces aires d'accueil devient donc une pratique illégale qui peut être sanctionnée. Ces sanctions ont été précisées en 2004 dans la loi de sécurité intérieure introduite par Nicolas Sarkozy, alors Ministre de l'intérieur. Les familles de Gens du voyage qui n'utilisent pas les aires d'accueil pour leur halte sont passibles, après avis d'expulsion :

- de six mois d'emprisonnement et une amende de 3750 euros (art. 322-4-1 du code pénal français) ;

- d'une suspension du permis de conduire pour une durée maximale de trois ans (art. 322-15-1 du code pénal français) ;

- de la confiscation du véhicule (art. 322-15-1 du code pénal français).

Notons ici le paradoxe de la confiscation du véhicule alors que ces sanctions visent, à l'origine, à régler le problème du stationnement illégal par le départ des familles vers des équipements adaptés et légaux.

\section{Le cercle vertueux des catégorisations : au-delà des catégorisations ethniques}

13 Au-delà de catégoriser en fonction de critères ethniques, la mobilité des familles est aussi sujette à de nombreuses classifications de la part des institutions françaises, démontrant, encore une fois, leurs soucis de mieux maîtriser la population. Elles opèrent notamment à différentes hiérarchisations des pratiques de mobilité pour organiser un accueil différencié au sein du territoire national. Ces catégories résultent de recensements spécifiques et d'enquêtes variées réalisés à différentes échelles pour comptabiliser les Gens du voyage en France. Cependant, la mobilité des familles complique considérablement toute tentative de dénombrement précis, rendant ainsi les données peu fiables et ouvertes à la critique notamment concernant les modalités de production des données. C'est pourquoi les principaux chiffres annoncés sont des estimations suivant une fourchette haute et basse. En France, la population des Gens du voyage est estimée entre 220000 et 250000 personnes (rapport Delevoye ${ }^{1}$ ). Dans ce rapport, une souscatégorisation est réalisée : les Gens du voyage sont "classés » selon leurs types de pratiques de mobilité spatiale. Ainsi, au début des années 2000, il y avait environ 70000 itinérants, 70000 semi-sédentaires et $110000 \mathrm{Gens}$ du voyage sédentaires. Le premier groupe représente les familles qui voyagent toute l'année, le second désigne les familles qui circulent une partie de l'année, souvent pendant la période estivale (ces familles interrompent notamment leur voyage durant la période scolaire), le dernier groupe correspond quant à lui aux personnes qui ont arrêté de voyager. Il est important de noter le caractère arbitraire de ces catégorisations qui figent les pratiques de mobilité des individus. Que faire des personnes considérées comme sédentaires (donc qui ne voyagent plus au moment du recensement ou de l'enquête) mais qui décident quelques semaines ou mois plus tard de reprendre le voyage? Le travail de terrain a notamment mis en 
évidence le caractère non figé des pratiques et de nombreuses familles ont repris une mobilité spatiale après une période plus ou moins longue de sédentarité. Certaines familles bricolent avec des périodes de mobilité ou de sédentarité à durée variable, ce qui rend totalement inappropriée cette classification.

Par ailleurs, nous pouvons observer que même si certaines familles arrêtent de voyager, beaucoup d'entre elles gardent un quotidien basé sur «l'expérience mobile » et sur la solidarité familiale. Elles continuent de vivre en caravane et les activités professionnelles sont aussi dépendantes d'un agenda axé sur la mobilité et la famille.

ces classifications, qu'elles soient réalisées en fonction d'un critère ethnique ou de types de pratiques de mobilité, ne correspond que très peu à la réalité du terrain que ce soit en termes de pratiques du voyage ou en termes d'identification collective et individuelle. En ce sens, mettre en regard ces catégorisations administratives avec celles opérées par les acteurs eux-mêmes est un travail très enrichissant qui permet d'identifier des décalages mais aussi de révéler un jeu de « bricolage identitaire ».

\section{Désignations endogènes}

16 L'analyse des désignations utilisées par les acteurs eux-mêmes dans leur vie quotidienne montre un réel décalage avec les catégorisations administratives officielles (Saul, Tebbut, 2005 ; Bergeon, 2011). Trois niveaux peuvent être identifiés notamment à travers l'observation des relations sociales.

Les familles n'utilisent que très rarement le terme officiel de "Gens du voyage ", à l'exception de situations administratives précises ou lors d'échanges avec les Gadjé ${ }^{2}$ (avec par exemple les acteurs associatifs ou encore lorsqu'ils se réfèrent à une aire d'accueil). $\mathrm{Au}$ quotidien, les Gens du voyage s'identifient comme des « Voyageurs », terme beaucoup plus signifiant pour les familles liées par une pratique du voyage valorisée et par un sentiment d'appartenance fort. Ce dernier est très largement revendiqué et contribue à créer un « sens collectif » dans un « ensemble populationnel » qui désigne « l'entité où se constitue, se cristallise et s'argumente du sens collectif [...] Ce sont les particularités des pratiques et des valeurs qui induisent sur la durée, le construit et la cohérence d'un ensemble populationnel » (Bouvier, 1995, p. 119).

L'unité familiale possède un rôle spécifique dans l'organisation quotidienne. Effectivement, la plupart des activités journalières (professionnelles, culturelles, religieuses, etc.) sont organisées en famille et l'ensemble des membres y participe. Un des exemples les plus significatifs concerne les activités économiques et les ressources financières qu'elles procurent. Celles-ci sont, le plus souvent, redistribuées au sein de l'ensemble de la famille afin que ses membres puissent en profiter. Les familles font preuve d'une grande souplesse et d'une grande malléabilité concernant les activités professionnelles. C'est d'ailleurs un atout considérable valorisé par les travailleurs sociaux qui œuvrent à l'insertion des Gens du voyage sur le marché du travail français. En plus de cette polyvalence, ils s'insèrent dans des niches économiques particulières comme celle de la récupération et du recyclage de la ferraille. À cela s'ajoutent des activités plus traditionnelles comme le rempaillage de chaises ou encore le cannage. Ils proposent souvent leurs services et leurs produits lors de marchés locaux ou nationaux et pratiquent le "porte-à-porte ». Aujourd'hui, la plupart des activités professionnelles traditionnelles exercées par les Gens du voyage ne sont pas reconnues par le marché légal du travail et ils sont touchés par un contexte économique de plus en plus régulé. C'est 
pourquoi l'un des premiers objectifs des structures sociales travaillant auprès des familles est de légaliser ces activités économiques, en particulier en les orientant vers l'autoentreprenariat. Cet exemple de la redistribution des ressources économiques à l'ensemble des membres illustre l'importance de la structure familiale dans la vie quotidienne. L'ensemble des témoignages récoltés lors du travail de terrain a souligné l'intérêt « d'être ensemble et de faire ensemble ». Cette proximité familiale se retrouve également dans les processus d'identification dans la communauté plus large des Gens du voyage où les réseaux familiaux - donc les alliances ou encore les conflits - sont clairement identifiés et revendiqués.

La dernière strate dans ces processus de désignation est la référence au « groupe culturel d'appartenance ». Si la recherche académique a très rapidement identifié divers groupes à l'intérieur même de la communauté des Gens du voyage, il en ressort, sur le terrain, que les références à ces différents groupes sont très récurrentes, montrant ainsi l'importance de se sentir appartenir à un groupe spécifique, avec des valeurs et des pratiques singulières. Ainsi, les Manouches, les Gitans, les Roms et les Yéniches illustrent la diversité de la communauté des Gens du voyage présente en France (Robert, 2007).

Afin de rendre compte de ce «bricolage identitaire ", la figure suivante représente les principales dénominations utilisées par les familles de Gens du voyage durant le travail de terrain.

Figure 1. Processus d'identification et sentiment d'appartenance.

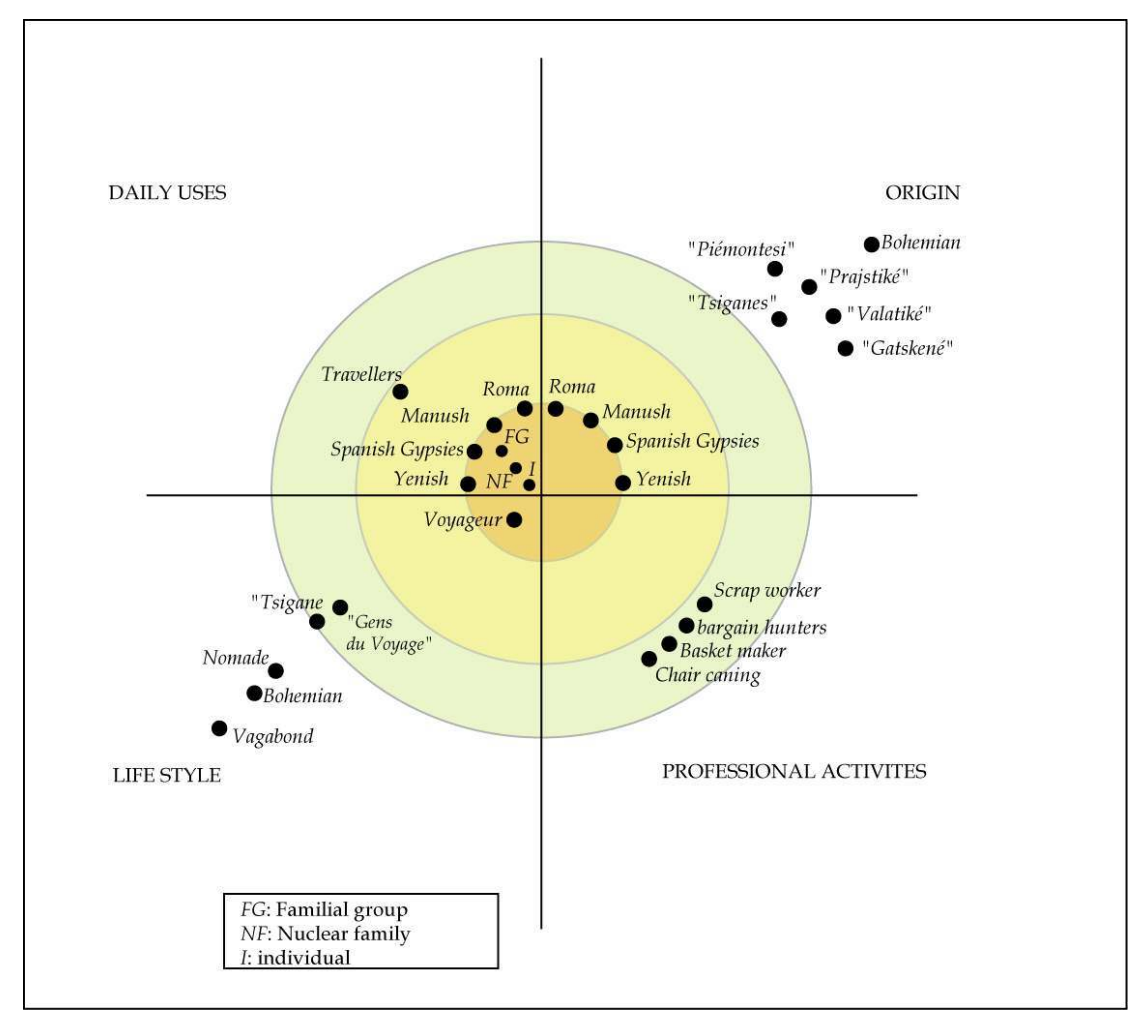

21 Ce schéma qui tente d'ordonner les appartenances multiples rencontrées au cours du travail de terrain ne résulte pas d'une enquête exclusivement centrée sur les divers types de désignations. Les différents termes utilisés lors des entretiens et lors des phases d'observation sur les aires d'accueil ont été spécifiquement notés. Les deux groupes culturels que forment les Roms et les Yéniches étaient globalement peu présents sur les 
différents lieux où s'est déroulée la recherche (voire totalement absents); ceci explique donc pourquoi ils sont peu représentés. Dans un même temps, ce schéma répartit l'usage des différentes désignations endogènes et exogènes. Plus le diamètre du cercle est important, plus le sentiment d'appartenance s'élargit. L'intensité de ce sentiment varie en fonction de ces strates. Les individus usent de leurs "casquettes" multiples et circulent dans les strates d'appartenance. Selon les expériences de vie et les rencontres, chaque individu mobilisera un capital social spécifique. Ce processus est souvent mentionné dans les études portant sur les minorités, comme celles de R. Kastoryano concernant la dynamique identitaire existant entre les minorités et la majorité « toute différence affichée, revendiquée et légitimée est source de paradoxe. Elle met en évidence une coexistence des différences comme mode de fonctionnement de la cité et engendre un même temps des conflits où chaque différence qui s'annonce, s'affirme et se confronte [...]. Bien entendu, toute relation dans l'altérité n'implique pas nécessairement le conflit, mais elle traduit de fait une relation de pouvoir qui génère souvent des conflits" (Kastoryano, 2005, p. 16). Ces va-et-vient identitaires décrivent un réel «bricolage des identités", mêlant références familiales et culturelles, de telle sorte que "lors des rencontres, chacun se juge et se jauge, s'identifie et se pose, se rattache à sa communauté familiale et rattache l'autre à la sienne, puis rapproche les familles entre elles, ou les distingue, et adopte un comportement de circonstance» (Liégeois, 2007, p. 56). Ce «bricolage » identitaire permet à l'individu de tempérer les processus d'exclusion à la fois à l'échelle de la famille et du groupe mais aussi au niveau du groupe culturel de référence, et plus généralement de la société globale.

\section{Les conséquences de la loi de 2000 sur les pratiques de mobilité des Gens du voyage}

Le paradoxe entre la présence historique des Gens du voyage et cette distance sociale opérée par la société française questionne leur reconnaissance et leur intégration au sein de la nation. Les politiques publiques récentes ne montrent pas d'évolution fondamentale concernant leur place dans la société. L'analyse des textes législatifs qui concerne le stationnement et l'accueil des Gens du voyage souligne une volonté réelle de réduire la mobilité des familles alors que les conditions de halte que ces textes proposent ont pour objectif premier le départ des familles de leur lieu de stationnement. Avant de présenter les dynamiques qu'engendre la loi en termes de nouvelles pratiques de mobilité, il est dans un premier temps important d'analyser les conséquences directes des dispositions législatives sur le voyage. Si nombre de familles rencontrées réussissent à réorganiser leur mobilité en fonction des contraintes opérées par les obligations de stationnement, d'autres ne parviennent pas à contourner ces obstacles.

\section{Des parcours désorganisés}

23 Une des principales conséquences des politiques publiques est la déstructuration des schémas familiaux de déplacement. Les parcours s'en trouvent souvent perturbés. En effet, les groupes possèdent des itinéraires identifiables dans l'espace et dans le temps; le parcours est réfléchi, alors que les familles sont contraintes, par la loi, de stationner sur des places identifiées et délimitées. Ainsi, certaines communes, habituellement investies par certaines familles ne sont pas dans l'obligation de créer un dispositif d'accueil: 
aucune possibilité d'arrêt légal n'existe alors pour répondre à la demande des familles. La seule solution qui s'offre à elles est de stationner sur des aires d'accueil officielles, souvent très éloignées du lieu initialement prévu. L'application de la loi de 2000 tend donc à modifier les parcours :

« Nous, ce qu'on aimait le plus, c'était qu'on pouvait s'arrêter là où on voulait. Je me souviens : mon père, un jour, on voulait aller au fond d'un chemin dans la forêt, et ben la caravane, la roulotte, ne passait pas : alors, mon père a tout coupé ; il a taillé les arbres pour qu'on passe. C'était vert, on pouvait marcher. Maintenant ... avec le goudron sur les aires d'accueil, moi, je serais malheureuse. » (Terrain Deux-Sèvres, 2007, Lali, sédentaire en terrain familial)

"Je me souviens bien. On savait exactement vers où aller. On savait de quoi vous aviez besoin. Même si l'image était difficile aussi... ce n'était pas pareil. Maintenant... on n'est plus à côté de vous, à votre proximité. On est là, sur les aires d'accueil. Et il faut qu'on se déplace loin pour aller vers le travail hein. C'est pareil pour les femmes. Tu sais que, en général, c'est elles qui font le porte-à-porte pour vendre les paniers. Sauf... bah... comment elles font maintenant, car elles n'ont pas le permis ?... Elles ne vont pas faire 10 kilomètres à pied, avec tous les paniers, pour aller aux habitations. Parfois on les amène et on revient les chercher plus tard... mais... ce n'est quand même pas facile... Pis, les jeunes, là, tu les as vus ? Ils ne savent plus travailler. » (John, Homme manouche, terrain Charente, 2008)

Les témoignages recueillis montrent que les aires d'accueil ont un réel impact sur les pratiques de mobilité, au point de les désorganiser. En effet, le législateur, en encadrant le stationnement - et donc la mobilité - provoque du désordre et une gêne dans la poursuite des parcours en orientant très concrètement les lieux et le temps de halte ${ }^{3}$.

Alors que le stationnement illégal engendre des sanctions sévères, les familles doivent composer également avec une pénurie d'aires d'accueil sur le territoire national. En effet, en 2013, environ $60 \%$ des recommandations mentionnées dans les schémas départementaux sont réalisés. L'obligation d'accueil n'est donc pas respectée par toutes les communes. C'est pourquoi un nombre considérable de familles sont continuellement en recherche de places libres pour leur halte. Cette pénurie instaure de fait une concurrence entre les familles de Gens du voyage. Certaines évoquent le terme «d'errance » pour définir ces déplacements qui n'entrent pas dans le cadre de leur parcours. Elles sont alors en « recherche de la légalité » et se retrouvent en quête d'un lieu d'arrêt légal. La mobilité devient balisée et orientée en fonction des espaces de stationnement libres. Du fait de cette mobilité orientée, les réseaux, qui sont d'habitude mobilisés dans les lieux clés du parcours, sont déconnectés et désorganisés. Les familles essaient alors au mieux de recréer du lien social, notamment pour exercer leurs activités professionnelles. Le manque d'aires de stationnement et l'inadéquation de celles-ci lorsqu'elles existent, sont les « aléas » de la loi de 2000.

\section{S'adapter à la loi et résister, développer de nouvelles pratiques de mobilité}

L'arrêt total de la mobilité a été le choix de nombreuses familles. Ce phénomène n'est pas seulement lié à la loi de 2000 mais aussi à un contexte économique de plus en plus contraignant comme nous l'avons déjà signalé. Avant les années 2000, cette tendance s'observait déjà et les ressources économiques liées à la mobilité et aux activités traditionnelles devenaient de plus en plus difficiles à acquérir dans un contexte d'accroissement des coûts liés à la mobilité (entretien des véhicules, carburant, etc.). 
Enfin, l'accès aux aides sociales, qui s'est considérablement débridé depuis la loi de 2000, constitue également un motif d'arrêt de la mobilité mentionné par les familles comme par les acteurs sociaux travaillant auprès des Gens du voyage.

Les familles qui voyagent encore aujourd'hui en France ont donc dû faire preuve d'adaptation et de stratégies pour réussir à perpétuer leur mode de vie.

Dans un premier temps, les rythmes de la mobilité ont été considérablement changés. Beaucoup de familles ont allongé leur durée de halte afin de rencontrer le moins de problèmes possibles quant à leur stationnement. Ces familles vivent sur les aires d'accueil durant la totalité de la durée autorisée ${ }^{4}$. Dans ce cas de figure, elles jouent sur la dimension temporelle sans changer les lieux de halte qui sont minutieusement sélectionnés en termes d'opportunités économiques. Les parcours restent multisites mais le nombre de lieux le composant est considérablement réduit.

Dans un second cas, c'est une accélération des rythmes quotidiens qui est entreprise. De nombreuses familles rencontrées ont fait l'acquisition d'un lieu de résidence stable et adaptent leurs déplacements caractérisés par d'intensifs allers et retours entre le domicile et les différents lieux de l'exercice de l'activité économique s'apparentant à des migrations pendulaires. Dans ce cas, le parcours est extensif et multilocal en référence aux travaux de M. Stock en 2006 concernant les modes de vie et les espaces vécus. L'intensification de la mobilité devient alors une ressource nouvelle (Morokvasiv-Muller, 1999).

\section{Être propriétaire pour mieux voyager : la stratégie de l'acquisition multisite}

$\mathrm{Au}$ cours de ces dernières années, nous pouvons observer un phénomène en forte croissance, celui de l'acquisition multisite. Il s'agit de terrains familiaux où les familles peuvent installer leurs caravanes sans contraintes (figure 2) et organiser leur vie quotidienne selon une organisation familiale resserrée, ce qui n'est que très rarement possible sur les aires d'accueil. En effet, sur les lieux de halte officiels fournis par l'Etat, une organisation stricte de la disposition des caravanes doit être respectée par les usagers, ce qui induit souvent un éloignement des familles dispersées sur l'ensemble de l'équipement. Sur ces terrains familiaux, comme nous pouvons le voir sur la figure 2, l'agencement des caravanes est réalisée de manière à créer un espace intime familial ; ou encore à l'extrémité du terrain, un espace est réservé à la récupération et au travail de la ferraille. Cet exemple d'organisation du stationnement, qui n'est pas un cas isolé dans le travail de terrain, est significatif à plusieurs points de vue : dans un premier temps, le lieu de stationnement est un réel lieu de vie où se déploient des activités quotidiennes et des solidarités familiales, ce qui est très rarement pris en considération dans la réalisation des aires d'accueil où la halte est considérée comme éphémère et dénuée de sens. Dans un second temps, cette tendance à l'acquisition multisite montre une inadéquation entre l'offre et la demande en termes d'agencement et d'équipements de lieux de halte officiels.

«Le tout, aujourd'hui, c'est de trouver un terrain. Et ça, c'est pas facile. Mais une fois qu'on a le terrain, déjà ça va mieux, on se sent quand même plus libres. Enfin, pour nous hein, parce que les aires d'accueil... soit c'est plein, soit c'est fermé, soit c'est mal placé... enfin, tu vois c'est pas possible. La chance qu'on a, nous, c'est qu'on a pas mal de cousins un peu partout - mais ça, c'est un peu tous les Voyageurs hein [rires] - et chez nos cousins, eh ben on peut quand même stationner. On retrouve un peu comme avant, pas au total mais disons qu'on se sent moins, moins, 
moins... plus libres!» (Terrain, Charente-Maritime, 2007, homme manouche propriétaire d'un terrain familial)

31 Ces familles deviennent donc propriétaires (très peu sont locataires de leur terrain familial) afin de faciliter le voyage. Replacée dans le contexte plus large de la circulation, c'est une réelle stratégie opérée par les familles qui vont acquérir, tout au long du parcours, de nombreux terrains familiaux et s'émanciper totalement des équipements offerts par l'Etat.

Figure 2. Organisation d'un terrain familial dans la Vienne (France).

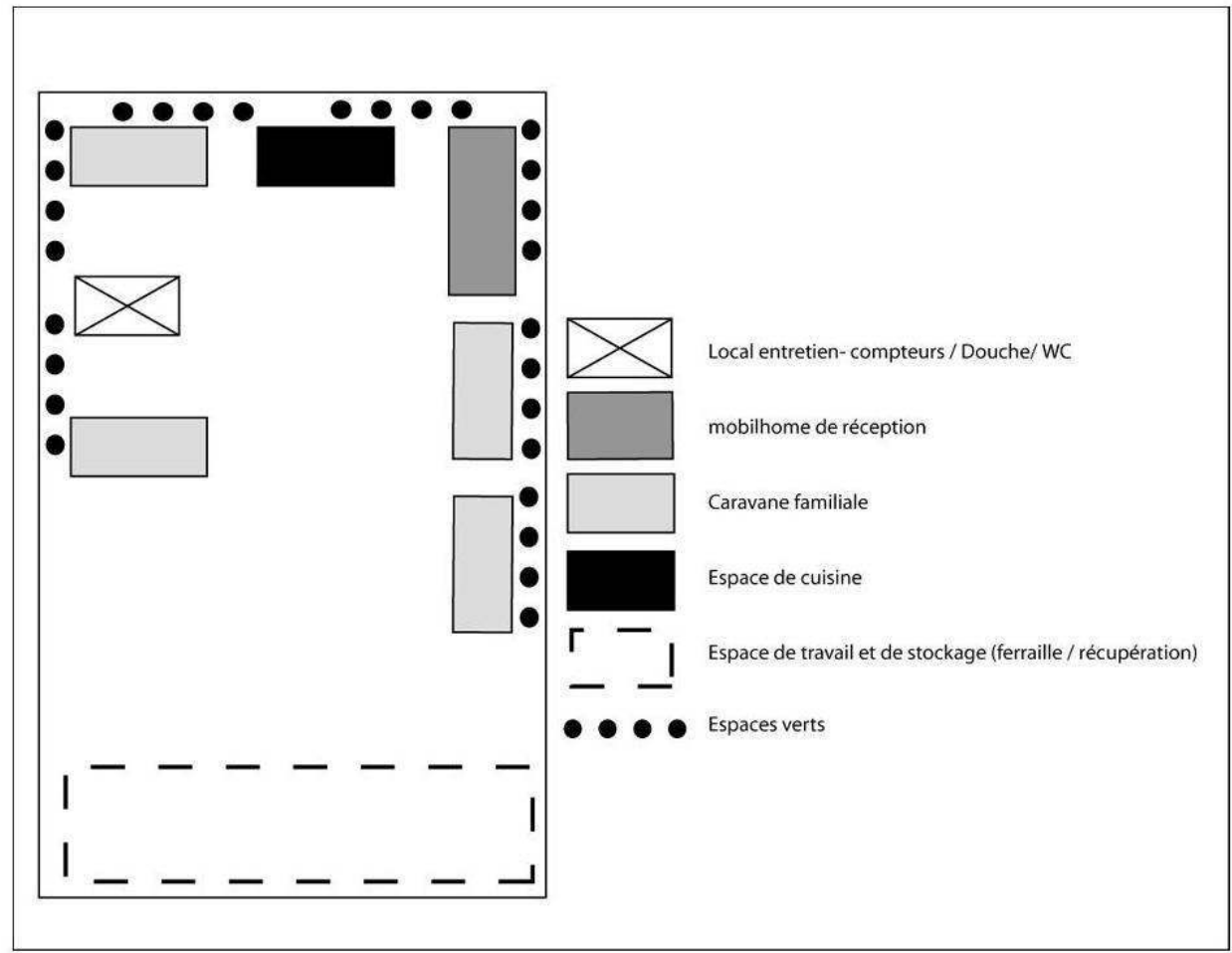

\section{La religion ou l'alibi de la mobilité}

La religion possède un poids considérable dans les logiques de mobilité actuelles, surtout pour les Gens du voyage de confession pentecôtiste et évangélique puisque ces Églises possèdent un culte sédentaire ainsi qu'un culte itinérant. Face aux gênes occasionnées par les politiques publiques de stationnement, la religion apparait comme une alternative possible pour les familles. Elle est considérée comme une solution à la circulation légiférée et offre aux familles en "mal de mobilité » d'autres possibilités de circuler. Le parcours comme la halte sont alors organisés par les pasteurs. L'itinérance religieuse structure donc la mobilité de nombreuses familles.

«- Ah oui, on participe à des grands rassemblements. En fait, maintenant, on commence à voyager quand les missions commencent. C'est très important et on se sent moins attaqués, vous comprenez. $Y$ en a qui vont commencer là bientôt, elles seront pas très loin, si vous voulez venir. On pourra vous informer des dates et des places. On fera passer le mot à $\mathrm{M}$. pour qu'il vous le dise.

- Et pourquoi vous y participez? Vous trouvez ça plus facile? 
- Disons que, déjà, c'est pour notre foi et puis, c'est vrai faut l'avouer, c'est quand même plus facile hein. Y a que comme ça qu'on voyage maintenant. Vous avez vu sur l'aire d'accueil, juste à côté, là, y a même un local de la mission Vie et Lumière. Ils nous accompagnent.» (Terrain, Deux-Sèvres, 2010, femme manouche "sédentarisée » en terrain familial mais sur les routes pendant la période des missions) pratiques de mobilités perdues ou devenues compliquées. Si les pasteurs ont trouvé de «bons fidèles » parmi les Gens du voyage, ils permettent aujourd'hui à de nombreuses familles d'éviter une «sédentarisation complète». L'autonomie, qui structure habituellement les pratiques de mobilité, est de fait amoindrie puisque ce sont les organisateurs des missions qui choisissent les dates et les lieux des rencontres. Le choix est donc restreint pour les familles, mais l'argument de la tranquillité de stationnement est souvent valorisé. Avec l'essor de la religion qui définit des pôles géographiques essentiels dans le parcours, le facteur économique s'amoindrit considérablement Ainsi, les familles ne circulent plus (ou moins) pour saisir des opportunités économiques rémunératrices, mais pour alimenter une pratique en perdition.

Cependant, les entretiens ont mis en évidence que l'itinéraire religieux, bien qu'il soit valorisé par les Voyageurs, n'est pas exempt de toute pratique économique. Ces réunions sont également un moyen d'établir de nouvelles alliances économiques. Par ailleurs, la participation de plus en plus massive des Gens du voyage (parfois plus de 2000 caravanes) augmente les possibilités de négociation entre les familles. Les forces de travail fusionnent pour un meilleur profit. Les réseaux économiques déjà préétablis - et souvent affaiblis - s'agrandissent et laissent la place à des opportunités plus grandes.

"J'me souviens d'un jour, c'était l'année où la Mimby [sa fille] commençait à fréquenter. On connaissait plus ou moins la famille. On sait tout, tu sais... et tu sais, les missions, c'est une ambiance bizarre mais agréable, on est tout le temps tous ensemble, y en a qui viennent et qui repartent. Dans l'autre famille, celle que fréquentait la Mimby, ils avaient repéré pas mal de ferraille chez un Gadjo. Ils y sont allés pour demander au Gadjo ce qu'il comptait faire de tout ça. Bref, au bout d'un moment, il a dit qu'on pouvait prendre, alors, ils sont venus me chercher, les hommes de l'autre famille... quand on est arrivés, mais c'est qu'il y en avait de la ferraille. Donc, on a tout fait ensemble et forcément ça a facilité les choses... [Rires].» (Terrain, 2007, Charente-Maritime, Manouche sédentaire, circulant pendant les missions)

\section{Conclusion}

Depuis le début des années 2000, les différentes mesures législatives qui ont été prises envers les Gens du voyage ont considérablement affecté la vie quotidienne et la mobilité des familles. Le voyage a subi de nombreuses modifications face à la volonté croissante de l'Etat de mieux contrôler leurs déplacements sur le territoire national. La création des aires d'accueil, de lieux uniquement dédiés aux Gens du voyage, n'a fait que renforcer l'image d'une population "problématique» et «à part» dans la société, comme le montrent d'ailleurs les différentes études annuelles du Comité National Consultatif des Droits de l'Homme. Du côté des familles, ces lieux, considérés comme des espaces clos, de parcage, les renvoient à une période sombre de leur histoire.

L'accueil proposé par les collectivités considère comme problématique la diversité interne à la population des Gens du voyage en termes de pratiques de mobilité comme en 
termes de groupes culturels de référence. Par ailleurs, cet accueil ne répond que partiellement à la demande des familles concernant l'agencement et les équipements des lieux de halte officiels. La loi de 2000 sur l'accueil et l'habitat des Gens du voyage impose aux communes de plus de 5000 habitants de créer des lieux de stationnement équipés qui, très souvent, les relèguent aux extrémités du territoire communal. Cet isolement ne fait que renforcer les dualités. Enfin, certaines communes doivent respecter la loi du fait de leur nombre d'habitants mais ne sont pas concernées par le passage et l'installation de familles de Gens du voyage, ce qui créé des équipements non utilisés. A l'opposé, des villes très fréquentées par les familles n'ont pas assez de capacités d'accueil. Ce constat montre le décalage existant entre l'offre et les lieux traditionnellement investis par les Gens du voyage. Les bassins de vie et de circulation des familles ne correspondent pas à l'organisation territoriale officielle.

Ces observations font résonance à l'analyse des motivations et des pratiques de mobilité qui, au contraire, révèle des parcours mûrement réfléchis qui prennent leurs sources dans des pratiques sur plusieurs générations de familles. Chaque lieu investi historiquement fait sens dans l'ensemble du parcours en termes de motivations familiales, religieuses ou encore économiques. Ces motivations constituent de réelles ressources sociales, culturelles et économiques et facilitent la poursuite de la mobilité (Bourdin, 2005). L'adoption de stratégies dans l'objectif de contrebalancer l'ordre imposé par l'Etat questionne l'évolution des lieux de halte officiels qui deviennent " plutôt qu'une invitation au voyage en parsemant la France d'étapes repérées ou adaptées, un lieu de contrôle, de regroupement surveillé d'une population-problème » (Charlemagne, 1983, p. 16).

Toutes ces mesures, mentionnées comme de réelles contraintes par les Gens du voyage, opèrent dans l'ensemble une modification de la mobilité et non sa disparition totale. Les pratiques ont été remodelées. Ces nouveaux «comportements circulatoires » (Bergeon, 2011) mettent en lumière les capacités d'adaptation des Gens du voyage et les dynamiques qui sont, petit à petit, mises en œuvre, pour répondre et " contourner » les dispositions législatives. Ces nouvelles pratiques ne sont pas identiques pour toutes les familles mais deviennent très régulières. Les familles sédentaires ne sont pas isolées et sont importantes à prendre en compte dans la mesure où elles participent considérablement à la continuité du voyage, pour ceux qui le pratiquent encore aujourd'hui. Elles ont un rôle majeur dans l'équilibre de la mobilité.

39 La place des Gens du voyage dans la société française a toujours été considérée comme problématique ainsi que nous l'avons vu avec l'instauration de toutes ces mesures spécifiques à leur encontre, notamment depuis le début du $20^{\text {ème }}$ siècle. Même si l'accès aux droits sociaux et économiques est en réelle amélioration, ces familles sont toujours touchées par des représentations négatives persistantes. Aujourd'hui, l'amalgame opéré par les institutions françaises entre les Gens du voyage français et les Roms migrants handicape fortement les premiers et contribue à maintenir un accueil hostile envers les Gens du voyage. 


\section{BIBLIOGRAPHIE}

BANCROFT A. (2001), « Closed spaces, restricted places. Marginalisation of Roma in Europe », Space and Polity, 5, 2, Glasgow, Department of Geographical and Earth Sciences, University of Glasgow, pp. 145-157.

BANCROFT A. (2005), Roma and Gypsy-Travellers in Europe: modernity, race, space and exclusion, Aldershot, Ashgate.

BERGEON C. (2011), Initiatives et stratégies spatiales. Le projet circulatoire face aux politiques publiques. L'exemple des Rroms et des Voyageurs du Poitou-Charentes (France) et de la Wallonie (Belgique), thèse de doctorat, Poitiers.

BLUM LE COAT J.Y., CATARINO C., QUIMINAL C. (2004), « Les Gens du Voyage : errance et prégnance des catégories ", in GOTMAN A., Villes et Hospitalités : les municipalités et les étrangers. Paris, Ed. de la Maison des Sciences de l'Homme, pp. 157-176.

BOEV I. (2008), Introduction au droit européen des minorités, Paris, L’Harmattan.

BOURDIN A. (2005), «L'individualisme à l'heure de la mobilité généralisée », in ALLEMAND S., ASCHER F., LÉVY J. (2004), Les sens du mouvement, Modernités et mobilité dans les sociétés urbaines contemporaines, Paris, Belin, pp. 91-98.

BOUVIER P. (1995), Socio-anthropologie du contemporain, Paris, Galilée.

FORMOSO B. (1987), Tsiganes et sédentaires : la reproduction sociale d'une société, Paris, L'Harmattan.

KASTORYANO R. (2005), Les codes de la différence: race, origine, religion, France, Allemagne, Etats-Unis, Condé-sur-Noireau, Sciences PO les Presses.

LIÉGEOIS J.P. (2009), Roms et Tsiganes, Paris, La Découverte.

MOROKVASIC-MULLER M. (1999), "La mobilité transnationale comme ressource : le cas des migrants de l'Europe de l'Est”, Cultures \& Conflit, 33-34, Paris, L'Harmattan, pp. 105-122.

OKELY J. (1983), The Traveller Gyspsies, Cambridge, Cambridge University Press.

PHILIPS D. (2010), « Minority ethnic segregation, Integration and Citizenship: a European perspective ", Journal of Ethnic and Migration Studies, 36, 2, Sussex, University of Sussex, pp. 209-225.

POWELL R. (2008), « Understanding the stigmatization of Gypsies: power and dialectics of (dis) identification », Housing, Theory and Society, 25, 2, Reading, University of Reading, pp. 87-109.

REYNIERS A. (1995), « Migrations, mouvements, identité », in Hommes et Migrations : Tsiganes et voyageurs, 1188-1189, Paris, Établissement public du Palais de la Porte Dorée - Cité nationale de l'histoire de l'immigration, pp. 45-51.

RICHARDSON J. (2006), « Talking about Gypsies: the notion of discourse as control », Housing Studies, 21, 1, Bristol, University of Bristol, pp. 77-96.

ROBERT C. (2007), Éternels étrangers de l'intérieur, Paris, Desclée de Brouwer.

SAUL N., TEBBUT S. (2005), The role of the Romanies: images and counter-images of « Gypsies »/ Romanies in European Union, Liverpool, Liverpool University Press. 
SIMHANDL K. (2006), « Western Gypsies and Travellers “Eastern Roma”: the creation of a political objects by the institution of the European Union ", Nations and Nationalism, 12, 1, London, Association for the Study of Ethnicity and Nationalism, pp. 97-115.

\section{NOTES}

1. Le rapport Delevoye a été commandé par le gouvernement dans l'objectif de créer la loi de 2000 sur l'accueil et l'habitat des Gens du voyage.

2. Le terme de Gadjé désigne ceux qui n'appartiennent pas à la communauté des Gens du voyage. Ce n'est pas un terme à connotation péjorative. Il signifie "celui qui vit là où il est né » en opposition à celui qui pratique le voyage.

3. Le stationnement sur les aires d'accueil implique de respecter un règlement intérieur qui précise notamment une durée maximale de halte dans le temps.

4. Cette durée peut varier de trois à neuf mois environ avec une possibilité de renouveler une fois la durée de la halte.

\section{RÉSUMÉS}

La catégorie administrative «Gens du voyage » est apparue dans les années 1990 en France pour désigner une population caractérisée par son mode de vie et par son type d'habitat. Elle recouvre l'ensemble des personnes dont l'habitat en résidence mobile est une pratique traditionnelle. Depuis le début du $20^{\text {ème }}$ siècle, les outils et les textes législatifs encadrant le statut et les déplacements des Gens du voyage se succèdent. Ces politiques publiques très restrictives ont des conséquences discriminatoires en termes de droits civils ou encore en termes de liberté de circulation sur le territoire français. L'ensemble de ces dispositifs juridiques a des répercussions sur le quotidien des familles ainsi que sur leur mobilité spatiale qui constitue encore, pour nombre d'entre elles, leur mode de vie. Nous présenterons plus spécifiquement les nouvelles stratégies de circulation opérées par les familles pour perpétuer leur mode de vie.

The administrative category of "Gens du voyage" appeared in the 1990s in France to identify a population characterised by a specific life style and a particular housing type. This category gathers all the people who live in mobile residence. Since the beginning of the $20^{\text {th }}$ century, different legislative tools were created to manage the statute and the mobility of Gypsies in France. These public policies are often restrictive and have discriminatory consequences in terms of civil rights or in terms of freedom of movement. All these legislative tools have implications on the daily life of Gypsies families and on their mobility. Amongst other observations, we especially want to explain the new strategies of mobility operated by the families in order to perpetuate their life style.

\section{INDEX}

Mots-clés : Gens du voyage, mobilité, politiques publiques, catégorisations, identité

Keywords : gypsies, mobility, public policies, identity 
AUTEUR

CÉLINE BERGEON

UMR 7301 Migrinter, Université de Poitiers, celine.bergeon@univ-poitiers.fr 